\title{
Long-term cancer surveillance: results from the Forteo Patient Registry Surveillance Study
}

\author{
A. Gilsenan ${ }^{1}$ (D) D. Harris ${ }^{1} \cdot$ M. Reynolds ${ }^{1} \cdot$ D. McSorley ${ }^{1} \cdot$ K. Midkiff $^{1} \cdot$ L. Jackson $^{1} \cdot$ B. Muldavin $^{2} \cdot$ N. Kellier-Steele $^{3}$. \\ E. Andrews ${ }^{1}$
}

Received: 17 July 2020 / Accepted: 26 October 2020 / Published online: 5 November 2020

(C) The Author(s) 2020

\begin{abstract}
Summary The Forteo Patient Registry estimated the incidence of osteosarcoma in US patients treated with teriparatide and enrolled in the study between 2009 and 2019. No incident cases of osteosarcoma were identified among patients registered, and the crude incidence rate was 0 (95\% confidence interval [CI], 0-10.2) cases per million person-years.

Purpose The prospective, voluntary Forteo Patient Registry was established to estimate the incidence of osteosarcoma in patients who have received treatment with teriparatide (Forteo).

Methods Information on US adults prescribed teriparatide and enrolled in the Forteo Patient Registry 2009-2019 was linked with data from participating state cancer registries annually (2010-2019) to identify incident osteosarcoma cases using a standardized linkage algorithm. Teriparatide exposure was ascertained from self-reported data that included teriparatide initiation and demographics necessary to complete linkage. Osteosarcoma cases diagnosed on or after January 1, 2009, were identified by participating state cancer registries. The crude incidence rate (IR) and standardized incidence ratio (SIR) of observed cases to the expected number of cases adjusted to the background rate (3 per million person-years) and corresponding 95\% CIs for the occurrence of osteosarcoma were calculated whereby the cumulative amount of person-time observed was adjusted for mortality. Results Data for 75,247 enrolled patients (representing 361,763 cumulative person-years) were linked to each of 42 participating state cancer registries (covering 93\% of the US population), which included information on 6180 cases of osteosarcoma. No matches with incident cases of osteosarcoma following registry enrollment were found. The crude IR was 0 (95\% CI, 0-10.2) cases per million person-years and the SIR was 0 (95\% CI, 0-3.0).

Conclusions The ability to draw conclusions about the incidence of osteosarcoma among patients participating in the registry was limited due to the smaller than expected amount of patient follow-up time and the fact that no cases were identified.
\end{abstract}

Keywords Cancer registry $\cdot$ Linkage $\cdot$ Osteosarcoma $\cdot$ Registry $\cdot$ Teriparatide

\section{Introduction}

Teriparatide (Forteo; Eli Lilly and Company) [1], first approved in November 2002 by the United States (US) Food and Drug Administration (FDA), is a recombinant human

A. Gilsenan

agilsenan@rti.org

1 RTI Health Solutions, Research Triangle Park, NC, USA

2 RTI International, Research Triangle Park, NC, USA

3 Eli Lilly and Company, Indianapolis, IN, USA parathyroid hormone analog indicated for the treatment of postmenopausal women with osteoporosis at high risk for fracture, and to increase bone mass in men with primary or hypogonadal osteoporosis at high risk for fracture. In July 2009, the indication for teriparatide was extended to include the treatment of osteoporosis associated with sustained systemic glucocorticoid therapy (glucocorticoid-induced osteoporosis) in both men and women.

In preclinical toxicology studies, teriparatide caused increases in bone mass and a dose-dependent increase in the incidence of osteosarcoma in rats [2]. Later animal studies identified a no-effect dose of $5 \mu \mathrm{g} / \mathrm{kg} /$ day in rats [3], and no osteosarcomas were observed in cynomolgus monkeys treated with this dose [4]. No cases of osteosarcoma emerged during 
clinical trials of teriparatide or in a 5-year posttreatment follow-up study, and few spontaneous cases of osteosarcoma have been reported in patients treated with teriparatide [1].

In 2009, the FDA required the implementation of a voluntary, prospective registry to estimate the incidence of osteosarcoma in patients receiving treatment with teriparatide as a condition for approval of teriparatide for the new glucocorticoid-induced osteoporosis indication [5]. The Forteo Patient Registry was established in the US in 2009 as a prospective voluntary registry to estimate the incidence of osteosarcoma in patients who have received treatment with teriparatide. This study is part of a broader postmarketing safety program evaluating the long-term safety of teriparatide using real-world data. Recently completed complementary studies have included the 15-year retrospective Osteosarcoma Surveillance Study [6], and a study using Medicare claims data to identify exposure and linkage with state cancer registries to identify osteosarcoma cases [7]. The methods of the Forteo Patient Registry have been described in detail elsewhere [8]. The aims of this paper are to present the final results of the Forteo Patient Registry in detail and to summarize the long-term safety evidence for teriparatide.

\section{Methods}

\section{Study overview}

Gilsenan et al. [8] described the methods of the Forteo Patient Registry in detail. Briefly, the Forteo Patient Registry was established in 2009 to estimate the incidence of osteosarcoma in US adult patients aged $\geq 18$ years who have used teriparatide. Patients enrolled in the registry voluntarily between 2009 and 2019. The information from all enrolled participants was linked annually (2010-2019) with all participating cancer registries to ascertain any incident cases of osteosarcoma (i.e., cases diagnosed after the date of starting teriparatide and after study enrollment). Patients were considered exposed to teriparatide if they self-reported use of the drug at least once. Patients were classified as new users if they initiated teriparatide less than 3 months from the date of registration, recent users if the teriparatide start date was 3 to 6 months before registration, and past users if they initiated more than 6 months before registration. An osteosarcoma case was defined as histologically confirmed osteosarcoma that produced osseous matrix and fell within one of the categories identified using International Classification of Diseases for Oncology, Third Edition.

The study was approved by the RTI International (RTI) institutional review board and by local institutional review boards affiliated with participating state cancer registries when required.

\section{Study size}

The intent of the study was to estimate the incidence of osteosarcoma in adult patients who received treatment with teriparatide by observing 1.7 million patient-years of followup time. If achieved, when compared with the background rate at study initiation of 2.7 cases per million population per year (95\% confidence interval [CI], 2.4-3.0; age-adjusted to the 2000 US standard population) [9], a threefold increase in the risk of osteosarcoma could be detected. A threefold risk increase would equate to an absolute risk increase of approximately one additional case per 185,000 patient-years observed.

\section{Patient recruitment and enrollment}

Patients were recruited primarily through pre-enrollment forms included in the teriparatide device packaging, treatment starter kits, and brochures distributed by physicians or nurses. Patients expressed interest in the registry via a completed preenrollment form. Eligible pre-enrolled patients were mailed a registration letter, registration form, informed consent form, and small compensation for their time in completing the forms. Registration was complete upon the patient's return of the completed forms.

\section{Cancer registry linkage}

Cancer registries in all 50 states and the District of Columbia were invited to participate in the Forteo Patient Registry.

Link Plus (version 2.0), a probabilistic matching software program available from the Centers for Disease Control and Prevention (CDC), was used to develop a standard linkage algorithm that was used by all participating state cancer registries to ensure the annual data linkage was performed consistently [10]. Only incident osteosarcoma cases were considered reportable study outcomes.

During the annual linkage process, cumulative registration data from all enrolled patients - including name, date of birth, address, telephone number, last four digits of their Social Security number, race, and ethnicity — were sent to participating state cancer registries via a secure file transfer protocol. State cancer registries linked the registration data locally with all incident osteosarcoma cases in adults diagnosed since January 1, 2009, in their state cancer registry database.

\section{Statistical analysis}

The incidence rate of osteosarcoma was calculated as the number of new osteosarcoma cases following initiation of teriparatide treatment divided by the cumulative personyears at risk adjusted for mortality, and an exact $95 \%$ CI for the incidence rate was provided. The cumulative person-time 
at risk of osteosarcoma for registered patients was estimated by multiplying the number of new patients in the registry each year by the number of years since they first took the drug and adjusted for mortality using age-specific mortality rates from the National Center for Health Statistics. A standardized incidence ratio (SIR) of observed cases to the expected number of osteosarcoma cases adjusted to the age and sex of teriparatide users ( 3 per million person-years) and corresponding exact 95\% CI was also calculated.

\section{Adverse event reporting}

The Forteo Patient Registry did not solicit adverse events (AEs) or product complaints; however, spontaneous reports of teriparatide AEs and/or product complaints from patients during the conduct of the study were reported to the teriparatide manufacturer as spontaneous AEs. Any osteosarcoma matches occurring after linkage with cancer registries were considered serious AEs and were reported to the teriparatide manufacturer.

\section{Results}

\section{Participant characteristics}

Among the 75,247 patients registered in the Forteo Patient Registry database, most were female $(88.6 \%)$, non-Hispanic (94.3\%), and white (93.9\%), and the mean age was 69 years (Table 1). Overall, $70.4 \%$ of registrants in the Forteo Patient Registry were "new users," defined as having a teriparatide

Table 1 Characteristics of patients included in the Forteo Patient Registry $(n=75,247)$

\begin{tabular}{lll}
\hline Characteristic & Category & Number $(\%)$ \\
\hline Age & Mean, 69 years (range, 18-104 years) & \\
Sex & Male & $8568(11.4)$ \\
& Female & $66,667(88.6)$ \\
& Unknown & $12(<0.1)$ \\
Race & White & $70,664(93.9)$ \\
& Black & $999(1.3)$ \\
& Asian & $1571(2.1)$ \\
& American Indian or Alaska Native & $241(0.3)$ \\
& Native Hawaiian & $15(<0.1)$ \\
& Other Pacific Islander & $40(<0.1)$ \\
& Other & $1296(1.7)$ \\
Ethnicity & Unknown & $421(0.6)$ \\
& Non-Hispanic & $70,927(94.3)$ \\
& Hispanic & $3139(4.2)$ \\
& Unknown & $1181(1.6)$ \\
\hline
\end{tabular}

start date less than 3 months from the date of registration; $18.4 \%$ of registered patients started teriparatide 3-6 months prior to completing registration, and $11.2 \%$ of registered patients started teriparatide more than 6 months before completing registration.

The overall sex distribution of patients who completed registration in the Forteo Patient Registry (89\% female, $11 \%$ male) was similar to the sex distribution of the general teriparatide user population (91\% female, $9 \%$ male) based on new patient start data [11]. Figure 1 displays the age distribution stratified by sex of the general teriparatide user population based on market research data, and Fig. 2 displays the distribution of age stratified by the sex of patients enrolled in the Forteo Patient Registry. As shown, the distribution of age by sex for those enrolled in the Forteo Patient Registry was generally similar to that of teriparatide users in the age category.

\section{Distribution by method of pre-enrollment and by geography}

The teriparatide packaging was the most common source of pre-enrollment forms received among registered patients, with other promotional materials the second most frequent source (Table 2). At least one patient from each participating state was included in the linkage file (Fig. 3), and the distribution of patients was consistent with the size of the population in each state. Although not all 50 states participated in the linkage, all patient data were provided to each of the participating state cancer registries in an effort to capture data on patients who may have moved across state lines after enrolling in the Forteo Patient Registry. States that did not participate in the study had a lower percentage of registered patients (i.e., $<1 \%$ or 1 to $<$ $5 \%$ of registered patients). Approximately $95 \%$ of all enrollees contained in the 2019 data linkage file lived in one of the 42 states (including the District of Columbia) that participated in the 2019 linkage.

\section{Linkage with cancer registries}

For the 10th and final annual linkage, completed September 25, 2019, data for 75,247 patients enrolled in the Forteo Patient Registry were linked to the research databases for each of the 42 participating state cancer registries (covering $93 \%$ of the US population). These registries included information on 6180 cases of osteosarcoma in adults aged 18 years or older diagnosed since January 1, 2009. Owing to the typical lag time of 9 to 18 months between date of diagnosis and the date cases are available in the cancer registry database, not all cases for more recent diagnosis years were available. On average, registries reported being mostly complete for diagnosis years 2009-2016, almost complete for diagnosis year 2017, 
Fig. 1 Age distribution among the general teriparatide population, stratified by sex (prevalent teriparatide users January 2009 through June 2018) $(n=75,247)$ (Adapted from IMS Health Prescription Data (LRx), January 2009-June 2018 data provided October 2018)

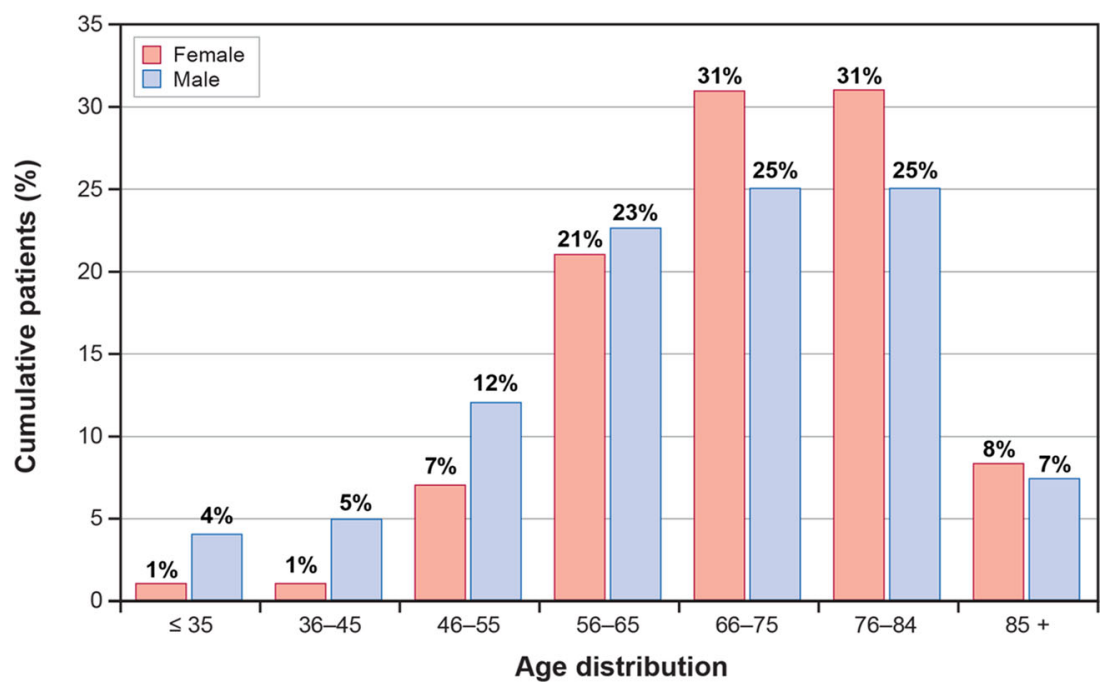

partially complete for diagnosis year 2018 , and only $2 \%$ complete for diagnosis year 2019.

No matches with incident cases of osteosarcoma following registry enrollment were identified during any of the 10 annual linkages with the cancer registries. The cumulative persontime at risk for registered patients was estimated at 361,763 person-years after adjusting for mortality; therefore, the crude incidence rate was 0 (95\% CI, 0-10.2) cases per million person-years. Compared with the expected number of cases adjusted to the background rate ( 3 cases per million personyears), the SIR was 0 (95\% CI, 0-3.0).

Of note, one case was identified during the seventh annual linkage in 2016 for a patient who had been diagnosed with osteosarcoma before enrolling in the Forteo Patient Registry. Because this case did not qualify as newly diagnosed after study enrollment, it was not included as a reportable study outcome. However, this case was reported to the FDA as a spontaneously identified AE.

\section{Discussion}

The Forteo Patient Registry enrolled over 75,000 patients during a 10 -year period and conducted annual linkages with individual cancer registries covering $93 \%$ of the US population. During this time, no incident cases of osteosarcoma were identified, although the likelihood of identifying one or more cases was somewhat limited given the lower than expected patient enrollment and cumulative person-years of followup. The fact that one case did match (despite not qualifying as a study outcome because the patient was diagnosed with osteosarcoma before enrolling in the Forteo Patient Registry) provides evidence that the linkage algorithm did work.

The Forteo Patient Registry is part of a broader postmarketing initiative to understand the long-term safety of teriparatide using real-world evidence. A complementary retrospective study, the Osteosarcoma Surveillance Study, identified incident cases of osteosarcoma through participating
Fig. 2 Age distribution of registered patients, stratified by sex, as of June 30, 2019. $N=$ 75,246 . One patient was missing data for sex and was excluded from counts in this figure

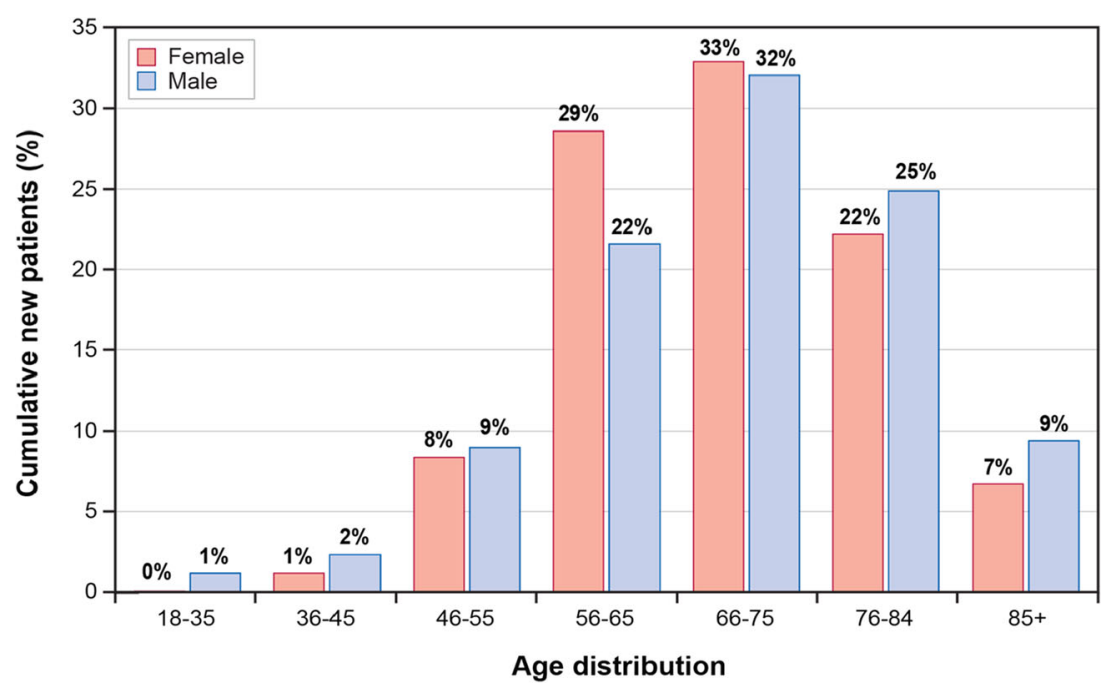


Table 2 Cumulative number of registered patients by mode of preenrollment

\begin{tabular}{ll}
\hline $\begin{array}{l}\text { Mode of pre-enrollment among registered } \\
\text { patients }(N=75,247)\end{array}$ & Number (\%) \\
\hline Packaging & $46,791(62)$ \\
Other promotional materials $^{\mathrm{a}}$ & $14,165(19)$ \\
Starter kit & $9868(13)$ \\
By phone & $2834(4)$ \\
Tear pad for physicians and nurse educators & $892(1)$ \\
Website & $697(1)$ \\
\hline
\end{tabular}

${ }^{a}$ Includes conversation tool, letters mailed from pharmacies, and teriparatide connect brochure

cancer registries in the US and evaluated potential teriparatide exposure through patient self-report or proxy report in telephone interviews [6]. Based on the background incidence rate for osteosarcoma, the expected number of cases among patients treated with teriparatide for this retrospective study was 4.17 , and 3 cases of osteosarcoma were observed among 1173 patients interviewed. This study's estimated standardized incidence ratio of expected to observed cases was $0.72(90 \% \mathrm{CI}$, $0.20-1.86$ ). In addition, a population-based comparative cohort study estimated the incidence rate ratio of osteosarcoma among US patients aged $\geq 65$ years treated with teriparatide (identified via Medicare prescription claims) versus a matched comparator cohort [7]. The incidence rate in the comparator cohort was consistent with the background incidence rate among adults aged $\geq 65$ years, and no osteosarcoma cases were observed in the teriparatide cohort, yielding an incidence rate ratio of 0 (95\% CI, 0-3.2).

Based on the totality of evidence from the Forteo Patient Registry, the Osteosarcoma Surveillance Study, and the Medicare comparative cohort study, none of which showed an increased risk of osteosarcoma in patients treated with teriparatide; the FDA released Eli Lilly, the manufacturer of teriparatide, from its commitment to continue this monitoring program [6,7]. Therefore, although the linkage of cumulative registration data from the Forteo Patient Registry had been planned to continue through 2024, the 2019 linkage was the final linkage of the study.

Some limitations of this study must be noted. First, the study did not have an internal comparator cohort, which was a key reason for initiating the Medicare comparative cohort study [7]. In addition, as with any voluntary registry, it is challenging to achieve sufficient participation, and the number of patients enrolled in the Forteo Patient Registry was insufficient to reach the target number of person-years at risk to evaluate the risk of developing osteosarcoma. Although no matches were found at the final cumulative 10th year of

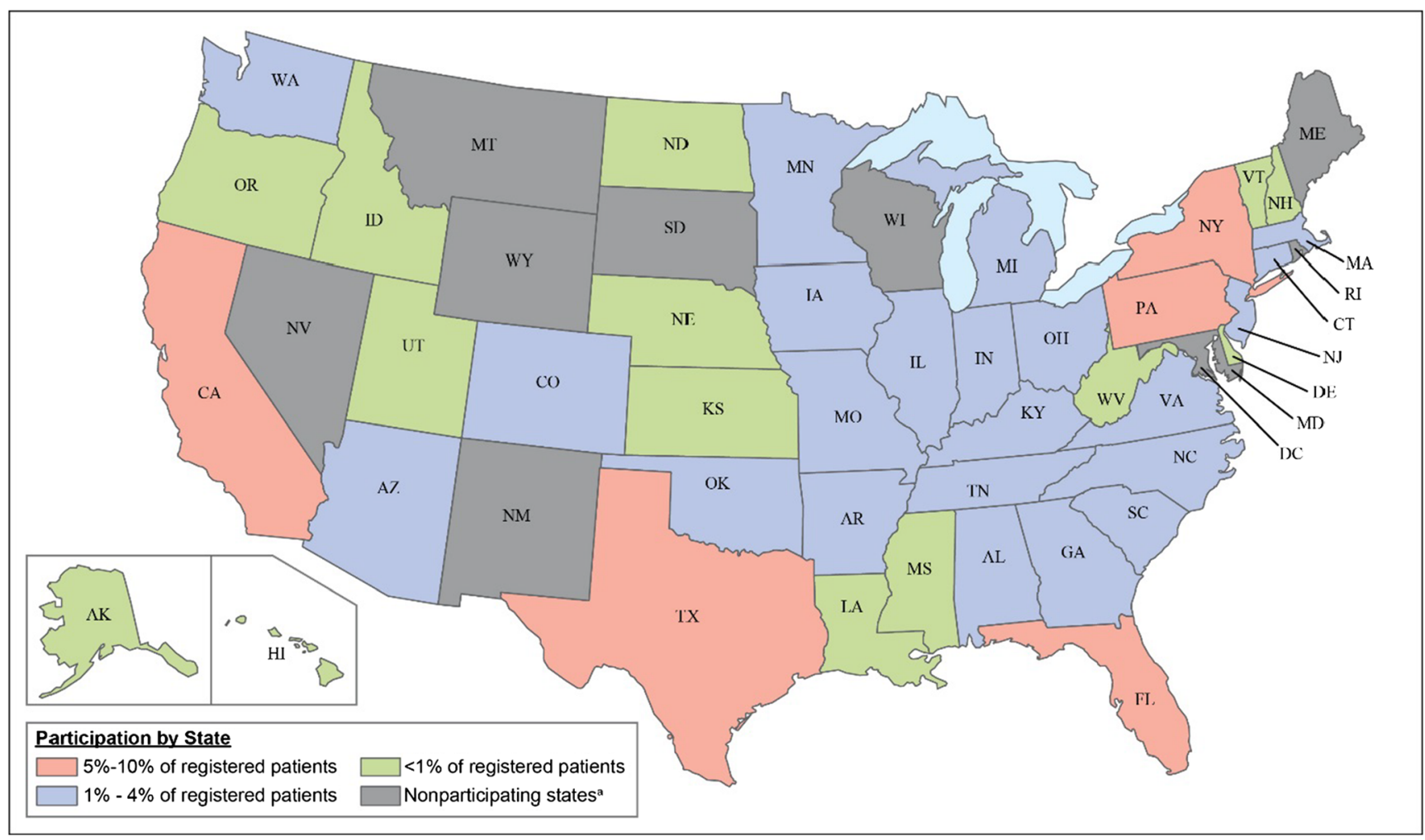

Fig. 3 Geographic distribution of patients included in the Forteo Patient Registry Linkage File $(n=75,247)$. (a) Approximately $5 \%$ of patients registered in the Forteo Patient Registry lived in one of the nine states where the cancer registry did not participate in the study. Geographic distribution based on state of residence at time of enrollment into the Forteo Patient Registry 
linkage, due to the low background rate of osteosarcoma, the $\mathrm{CI}$ of the final rate of osteosarcoma ( 0 to 10.2 cases per million person-years) is wide. Despite the imprecision, the upper bound of the $95 \%$ CI for zero cases is still a very low incidence. Importantly, no information on total duration of teriparatide treatment or other clinical information between time of registration and linkage with the cancer registries was collected. The simplified patient recruitment process required trade-offs between increasing participation and quantity of data collected. Such information would have been useful for subset analyses if the study had generated a higher incidence of osteosarcoma among teriparatide users than the general population, which was not the case. Further, due to the voluntary nature of the study, the group of patients who participated in the registry was not a random sample of the overall teriparatide patient population. It is possible but not likely that factors associated with the development of osteosarcoma could be differentially distributed between participants and nonparticipants. Thus, the incidence estimate based on participation may be biased. The analytic approach used in the study did not address the lag period between the date of cancer diagnosis and availability of patient data in the statewide cancer registries; the overall lag period between diagnosis and availability of data ranges from 9 to 18 months. Furthermore, given that the latency of osteosarcoma is unknown, this study does not address a possible extended latency period for development of osteosarcoma. Finally, due to the voluntary nature of the registry, generalizability to all patients treated with teriparatide should be made with caution. The comparison of available characteristics suggests that those who enrolled in the Forteo Patient Registry were similar to patients who were prescribed teriparatide; however, unmeasured characteristics that could potentially predispose (or not) patients to osteosarcoma could make results less generalizable.

\section{Conclusions}

The ability to draw conclusions about the incidence of osteosarcoma among patients participating in the registry was limited due to lower than expected patients enrolled and the smaller than expected cumulative amount of patient followup time, as well as the fact that no cases were identified. Given the SIR comparing the observed number of cases to the expected number of cases based on the adjusted background rate of 3 per million person-years, the findings suggest there could be somewhere between no increased risk of osteosarcoma up to a 3 -fold increase.

Acknowledgments The following 42 individual state cancer registries and their departments of health utilized their cancer incidence data for linkage in this study: Alabama Department of Health, Alabama Statewide
Cancer Registry; Alaska Department of Health and Social Services, Alaska Cancer Registry; Arizona Department of Health Services, Arizona Cancer Registry; Arkansas Department of Health, Arkansas Central Cancer Registry; California Department of Public Health, California Cancer Registry; Colorado Department of Public Health and Environment, Colorado Central Cancer Registry; Connecticut Department of Public Health, Connecticut Tumor Registry; District of Columbia Department of Public Health, District of Columbia Cancer Registry; Delaware Health and Social Services Division of Public Health, Delaware Cancer Registry; Florida Department of Health, Florida Cancer Data System; Georgia Department of Public Health, Georgia Cancer Registry; University of Hawaii Cancer Center, Hawaii State Department of Health, Hawaii Tumor Registry; Idaho Department of Health and Welfare, Cancer Data Registry of Idaho; Illinois Department of Health, Illinois State Cancer Registry; Indiana State Department of Health, Indiana Cancer Registry; University of Iowa, State Health Registry of Iowa; Kansas Department of Health and Environment, Kansas Cancer Registry; University of Kentucky, Kentucky Cancer Registry; Louisiana State University, Louisiana Tumor Registry; Massachusetts Department of Public Health, Massachusetts Cancer Registry; Michigan Department of Community Health, Michigan Cancer Surveillance Program; Minnesota Department of Health, Minnesota Cancer Surveillance System; University of Missouri, Missouri Cancer Registry; Nebraska Department of Health and Human Services, Nebraska Cancer Registry; New Hampshire Department of Health and Human Services, New Hampshire Cancer Registry; New Jersey Department of Health, New Jersey State Cancer Registry; New York State Department of Health, New York State Cancer Registry; North Carolina Department of Health and Human Services, Division of Public Health, North Carolina Central Cancer Registry; North Dakota Department of Health, North Dakota Statewide Cancer Registry; Ohio Department of Health, Ohio Cancer Incidence Surveillance System; Oklahoma State Department of Health, Oklahoma Central Cancer Registry; Oregon Department of Human Services, Oregon State Cancer Registry; Pennsylvania Department of Health, Pennsylvania Cancer Registry; South Carolina Department of Health and Environmental Control, South Carolina Central Cancer Registry; Tennessee Department of Health, Tennessee Cancer Registry; Texas Department of State Health Services, Texas Cancer Registry; Utah Department of Health, University of Utah, Utah Cancer Registry; Vermont Department of Health, Vermont Cancer Registry; Virginia Department of Health, Virginia Cancer Registry; Washington State Department of Health, Washington State Cancer Registry; West Virginia Department of Health and Human Services, West Virginia Cancer Registry; the Surveillance, Epidemiology, and End Results (SEER) Program of the National Cancer Institute; and the National Program of Cancer Registries of the CDC. Use of these data does not imply that these registries, their departments of health, the CDC, or SEER either agrees or disagrees with any presentations, analyses, interpretations, or conclusions. The authors acknowledge and thank the GHBX Advisory Board members and guests of the board who have provided thoughtful input throughout the conduct of this study. Advisory board members include Dr. Bruce Chabner from Harvard Medical School; Prof. Henrik Bauer from Karolinska Institute; and Dr. A. Kevin Raymond, formerly from MD Anderson. Guests of the board, Dr. Maria Schymura, Dr. Kenneth Rothman, and Dr. Thor Alvegard, also provided valuable insight and advice. The authors gratefully acknowledge the following people for their contributions to this study: Abenah Harding and Diana Goss of RTI Health Solutions and the many members of the project team working from RTI International's Research Operations Center, including Lynda Tatum, Jeremiah Robinson, Raquel Taylor, Michael Todd Prince, Pat Brunson, Sabrina Burgos, Adam Kaderabek, Verna Barnhill, and Cheryl Williams. Kate Lothman of RTI Health Solutions provided medical writing services, which were funded by Eli Lilly and Company. 
Funding This study was funded by Eli Lilly and Company.

\section{Compliance with ethical standards}

Conflicts of interest This study was performed under a research contract between RTI Health Solutions and Eli Lilly and Company and was funded by Eli Lilly and Company. Elizabeth Andrews, Alicia Gilsenan, David Harris, David McSorley, Kirk Midkiff, and Maria Reynolds are salaried employees of RTI International, a nonprofit research organization that conducts research with multiple pharmaceutical companies and has an independent right to publish the results of this study. Nicole KellierSteele is a salaried employee of Eli Lilly and Company.

Consent to participate All patients participating in this study provided informed consent.

Open Access This article is licensed under a Creative Commons Attribution-NonCommercial 4.0 International License, which permits any non-commercial use, sharing, adaptation, distribution and reproduction in any medium or format, as long as you give appropriate credit to the original author(s) and the source, provide a link to the Creative Commons licence, and indicate if changes were made. The images or other third party material in this article are included in the article's Creative Commons licence, unless indicated otherwise in a credit line to the material. If material is not included in the article's Creative Commons licence and your intended use is not permitted by statutory regulation or exceeds the permitted use, you will need to obtain permission directly from the copyright holder. To view a copy of this licence, visit http:// creativecommons.org/licenses/by-nc/4.0/.

\section{References}

1. Eli Lilly and Company (Revised 2020) Forteo [package insert]. http://www.accessdata.fda.gov/drugsatfda_docs/label/2009/ 021318s012lbl.pdf. Accessed 11 Jun 2020

2. Vahle JL, Sato M, Long GG, Young JK, Francis PC, Engelhardt JA, Westmore MS, Ma YL, Nold JB (2002) Skeletal changes in rats given daily subcutaneous injections of recombinant human parathyroid hormone (1-34) for 2 years and relevance to human safety. Toxicol Pathol 30:312-321
3. Vahle JL, Long GG, Sandusky G, Westmore M, Ma YL, Sato M (2004) Bone neoplasms in F344 rats given teriparatide [rhPTH(134)] are dependent on duration of treatment and dose. Toxicol Pathol 32:426-438

4. Vahle JL, Zuehlke U, Schmidt A, Westmore M, Chen P, Sato M (2008) Lack of bone neoplasms and persistence of bone efficacy in cynomolgus macaques after long-term treatment with teriparatide [rhPTH(1-34)]. J Bone Miner Res 23:2033-2039

5. Food and Drug Administration (2009) Approval letter: Forteo (teriparatide, $\mathrm{rPTH}[1-34]$ ) for the treatment of men and women with osteoporosis associated with sustained systemic glucocorticoid therapy at high risk for fracture. https://www.accessdata.fda. gov/drugsatfda_docs/appletter/2009/021318s012ltr.pdf. Accessed 28 Sept 2017

6. Gilsenan A, Midkiff K, Harris D, Kellier-Steele N, McSorley D, Andrews E (forthcoming) Teriparatide did not increase adult osteosarcoma incidence in a 15 -year US postmarketing surveillance study. J Bone Miner Res

7. Gilsenan A, Midkiff K, Harris D, McQuay L, Hunter S, KellierSteele N, Andrews E (2020) Assessing the incidence of osteosarcoma among teriparatide users based on Medicare Part D and US State Cancer Registry Data. Pharmacoepidemiol Drug Saf. https:// doi.org/10.1002/pds.5103

8. Gilsenan A, Harding A, Kellier-Steele N, Harris D, Midkiff K, Andrews E (2018) The Forteo Patient Registry linkage to multiple state cancer registries: study design and results from the first 8 years. Osteoporos Int 29(10):2335-2343

9. Surveillance, Epidemiology, and End Results (SEER) Program (Released April 2006, based on the November 2005 submission) SEER*Stat Database: Incidence - SEER 17 Regs Public-Use, Nov 2005 Sub (2000-2003), National Cancer Institute, DCCPS, Surveillance Research Program, Cancer Statistics Branch. http:// www.seer.cancer.gov. Accessed 12 Jun 2020

10. Harris DH, Midkiff K, Gilsenan A, Andrews E (2010) Public health surveillance collaboration: establishing a linkage algorithm with cancer registries for the Forteo Patient Registry. Poster presented at the North American Association of Central Cancer Registries Annual Conference; June 2010. Quebec City, Quebec, Canada

11. IMS Health Prescription Data (LRx), January 2009-June 2018 data provided October 2018

Publisher's note Springer Nature remains neutral with regard to jurisdictional claims in published maps and institutional affiliations. 